\title{
INVESTIGACIONES
}

\section{Profesores principiantes de Educación Básica: dificultades de la enseñanza en contextos escolares diversos}

\author{
Novice teachers in primary education: difficulties of teaching \\ in diverse school's contexts
}

Tatiana Elena Cisternas León

CIDE (Centro de Investigación y Desarrollo en Educación)

Facultad de Educación, Universidad Alberto Hurtado

Correo electrónico: tcistern@uahurtado.cl

\begin{abstract}
RESUMEN
Este artículo presenta una selección hallazgos producto de dos investigaciones enmarcadas en una línea de trabajo que estudia la iniciación profesional en docentes principiantes. Ambos estudios -de naturaleza cualitativa- centran su interés en las dificultades percibidas por profesoras y profesores de educación básica durante sus primeros años de iniciación y explora el modo en que los contextos escolares acogen a estos jóvenes profesores en sus comienzos, facilitando u obstaculizando un periodo que se sabe es crítico para la configuración de identidad y el fortalecimiento de la vocación profesional. Los resultados muestran variaciones, más que un patrón evolutivo y homogéneo, asociadas a las oportunidades que la formación ofreció para problematizar el aula y el trabajo docente y a establecimientos escolares flexibles o burocráticos, que apoyan o presionan. Se concluye en torno la necesidad de diseñar programas de inducción y mentoría desde una perspectiva contextualizada, diversificada, evitando homogeneizar necesidades.
\end{abstract}

Palabras clave: profesores principiantes, formación docente, dificultades en la enseñanza, inserción profesional, contexto escolar

\section{ABSTRACT}

This article presents a selection of findings obtained in two investigations from a research which studies the first period of working practice of recently graduated teachers. Both qualitative studies focus on the difficulties perceived by teachers from primary education during their first years as professionals, and explore the way how school contexts receive young new teachers facilitating or holding up their performance in a critical period of identity construction and strengthening of the vocation. Results show variations rather than a developmental and homogeneous pattern; this is associated with the opportunities offered by the teaching formation in terms of questioning the classroom and the teaching performance, and the schools as either flexible or bureaucratic supporting or putting pressure. The study concludes by posing the need to design induction and mentoring programs from a contextualized and diversified perspective, avoiding the homogenization of teachers' needs.

Key words: beginning teachers, teacher education, concerns about teaching, teaching 


\section{INTRODUCCIÓN}

Este artículo presenta una selección de hallazgos producto de dos investigaciones enmarcadas en una línea de trabajo que busca profundizar el conocimiento sobre el período de iniciación profesional en docentes principiantes de Educación Básica ${ }^{1}$. Ambos estudios -de naturaleza cualitativa- centran su interés en las dificultades experimentadas por profesoras y profesores de educación básica, durante su primer año de iniciación, y explora el modo en que los contextos escolares acogen a estos jóvenes profesores en sus comienzos, facilitando u obstaculizando un período que se sabe es crítico para la configuración de la identidad y el fortalecimiento de la vocación profesional. Asimismo, la literatura en este campo ha señalado con fuerza la relación de interdependencia que existe entre las dificultades de la iniciación y el modo en que la escuela percibe y recibe al nuevo profesor. De allí que en este trabajo se ofrezcan elementos para comprender la diversidad de preocupaciones sobre la enseñanza sentidas por los propios docentes principiantes y las características de los entornos laborales donde ellos se insertan.

Detenerse en este actor es clave no solo para avanzar en el conocimiento sobre las necesidades y los soportes idóneos y pertinentes a nuestra realidad educativa, sino también porque informa acerca de las articulaciones (o brechas) entre la formación inicial y el desarrollo profesional, en tanto proceso de construcción y consolidación de identidades docentes. Además, contribuye con información relevante a la formulación de políticas locales y nacionales de mentoría e inducción en el marco de las nuevas exigencias de la carrera docente.

$\mathrm{El}$ artículo se estructura en base a cinco apartados. En primer lugar, se presenta la problematización que enmarca ambos estudios y algunos elementos conceptuales que orientan el desarrollo de estos trabajos. Luego, se describen objetivos y la estrategia metodológica utilizada. Ambas investigaciones utilizan un diseño de Estudio de Casos y procedimientos de análisis que siguen algunos principios de la Teoría Fundamentada. En el tercer apartado se exponen los hallazgos vinculados a las dificultades en torno a la enseñanza que perciben los docentes en su primer año laboral. En el cuarto apartado se analiza aquello que los docentes principiantes describen sobre sus contextos de iniciación y las estrategias de apoyo y seguimiento de las que son objeto. En una quinta parte concluimos respecto de la naturaleza de estas dificultades y el modo en que los entornos escolares de iniciación pueden convertirse en fuente de apoyo u obstáculo para hacer frente a estos desafíos. Además, se ofrecen recomendaciones para el diseño de políticas educativas orientadas al profesorado en este período de su desarrollo profesional.

\section{PROBLEMÁTICA Y ALGUNOS ELEMENTOS CONCEPTUALES}

La iniciación profesional supone un proceso de transición desde profesor en formación hacia un profesional autónomo, como parte de un continuo en el proceso de desarrollo

La primera investigación se enmarca en el trabajo realizado desde la Sub-línea de Formación Inicial Docente financiada por CEPPE: Dificultades experimentadas por profesores principiantes de Educación Básica en sus prácticas de enseñanza y su relación con la formación inicial.

La segunda investigación contó con el apoyo de CONICYT, Programa Fondecyt de Iniciación. Proyecto $\mathrm{N}^{\circ} 11130279$ :

Diferencias en los procesos de inserción a la docencia en escuelas chilenas. Los efectos de distintos contextos escolares de iniciación sobre prácticas de enseñanza, incidentes críticos y estrategias en profesores principiantes. 
caracterizado por ser "un período de tensiones y aprendizajes intensivos en contextos generalmente desconocidos y durante el cual los profesores principiantes deben adquirir conocimiento profesional además de conseguir mantener un cierto equilibrio personal" (Marcelo, 2009: 5). En efecto, investigaciones sobre los ciclos profesionales del profesor destacan el impacto que generan las primeras experiencias sobre sus creencias y sus prácticas pedagógicas (Goodson \& Heragraves, 1996; Day \& Gu, 2012). Esto, ya que se trataría de un proceso que no es lineal ni unidimensional, sino más bien complejo de caracterizar, pues se constituye al menos en base a cuatro elementos: a) las características de la formación inicial; b) las características de los contextos escolares donde se inserta el principiante y c) las formas de apoyo y la presencia o ausencia de apoyos que "suavicen" el proceso y d) actitudes y condiciones personales y biográficas del propio docente (Cornejo, 1999; Day \& Gu, 2012; Flores, 2008; Fuentealba, 2006; Feiman-Nemser, 2001; Huberman, 1989; Marcelo, 1988, 2008).

Además, existe consenso acerca de la relevancia de esta fase de iniciación, por cuanto este constituye un eslabón fundamental del desarrollo profesional docente y su estudio contribuiría con insumos relevantes al mejoramiento tanto de la formación inicial y continua de profesores, pero más especialmente, permitiría formular programas y políticas de apoyo a los procesos de inserción que vive el profesor principiante (Ávalos, 2011; Borko, 2004; Cornejo 1999; Day 2008; Feiman-Nemser 2001; Flores, 2008; Fuentealba, 2006; Ingersoll \& Strong, 2011; Marcelo, 2008; Marcelo, 2009; Kensigton, 2011; Tynjälä y Heikkinen, 2011).

El conjunto de la investigación sobre los primeros años de la enseñanza se ha orientado hacia cuatro vertientes fundamentales: a) los dilemas y conflictos que experimentan los profesores principiantes, b) su inserción como un proceso de socialización; c) la construcción de identidad docente, y d) los apoyos que recibe el docente en este período, por medio de iniciativas formales como los programas de inducción e informales o tácitas desarrolladas por la propia institución escolar ante la llegada de un docente novato (Flores, 2008).

Dentro de este campo, diversos trabajos muestran cómo el profesor vive un choque con la realidad que se expresa en dificultades, preocupaciones y dilemas de diversa naturaleza a los cuales debe hacer frente con recursos también variables. Estas preocupaciones pueden agruparse en dos niveles. Por una parte, el macronivel, referido a problemas ligados a la organización escolar (relación con colegas, directivos, apoderados) y asuntos asociados a un micronivel, es decir vinculado a las decisiones de aula: contenidos, materiales, organización de la clase, gestión de los alumnos, etc. (Marcelo, 1988; Vonk \& Schras, 1987). Este último ámbito ha comenzado a ser estudiado más recientemente desde una dimensión pedagógica y didáctica, es decir, enfocada en dilemas, estrategias de abordaje e instancias de apoyo vinculadas a las tareas enseñanza-aprendizaje en el aula. Más aún, pasados los primeros meses de inserción, los problemas que viven los principiantes se concentran en el campo de las prácticas de enseñanza (Day, 2008; Lam, 2011; Long, 2012; Tynjälä \& Heikkinen, 2011; Flores, 2015; Agüero 2015; Rufinelli, 2014, Flores, 2014).

Desde esta perspectiva, algunas investigaciones destacan el valor que alcanzan ciertos dispositivos de apoyo para generar procesos más exitosos de inserción en la docencia (Borko, 2004; Ingersoll \& Strong, 2011; Meijer, Korthagen \& Vasalos, 2009). Diferentes oportunidades para enfrentar tareas vinculadas a la enseñanza hacen una diferencia, "mitigando" los procesos de incertidumbre y shock que viven habitualmente los principiantes (Orland-Barak, 2008: 166-167). Otros estudios señalan que la autoeficacia mejora considerablemente en futuros profesores que realizaron prácticas en contextos difíciles (Rushtton, 2001). 
En Chile, hemos visto un interés creciente por este actor, gracias al impulso que ha tenido en los últimos años temas como la inducción a la docencia, las mentorías y el seguimiento a los egresados (González, Araneda, Hernández \& Lorca, 2005; Boerr, 2011; Cisternas, 2011). Investigaciones a estudiantes en práctica profesional y profesores principiantes señalan la poca contribución que tuvo la formación inicial para resolver situaciones típicas de la enseñanza, por ejemplo, diseñar un instrumento de evaluación que diese cuenta de la diversidad de aprendizajes en el aula (Ávalos, 2005; Flores, 2014; Montecinos, Walker, Solís, Núñez, Contreras \& Rittershaussen, 2010; Peralta, 2009; Ruffinelli, 2013, 2014).

Por otra parte, la literatura más reciente sobre docentes novatos ha reforzado la comprensión de las experiencias de los profesores noveles a partir de lo que ocurre en las escuelas donde ejercen. Los investigadores han argumentado que la calidad del ambiente de la escuela es tal vez el factor más importante que influye en la crecimiento y desarrollo profesional de los profesores noveles (Day, 2006, 2008; Giffin et al., 2009). Algunos autores destacan que, según el contexto donde se inicie, el joven docente se enfrentaría a dos precariedades: por una parte, una formación inicial docente poco pertinente y, por otra, a contextos profesionales con tendencia al trabajo individual y rutinario que generan un aprendizaje vicario que descarta innovación (Imbernón, 1994). Otras investigaciones señalan que la superación de los problemas se realiza en forma personal e idiosincrática (Flores, 2008), dependiendo asimismo de factores personales y de las culturas organizacionales en las cuales se insertan (Marcelo, 1991). En Chile, este escenario cobra mayor actualidad, ya que como lo han mostrado algunas investigaciones, la mayoría de los docentes que se desempeñan en el sistema escolar subvencionado no tienen una inserción mediada; al contrario, se inician con una carga de trabajo igual o superior a la de un docente experimentado y se le exige tanto o más que aquel (Avalos, Carlson \& Aylwin, 2004; Peralta, 2009).

En suma, indagar sobre los procesos de iniciación a la enseñanza del profesor principiante y los contextos donde se inserta, supone entrar en el campo del desarrollo profesional docente en la medida en que los dilemas, las estrategias y las propias prácticas permiten reconocer y caracterizar esta fase de la vida del docente. Si bien hay cierta claridad sobre las dificultades más habituales que vive el principiante, cabe preguntarse más específicamente por aquellas vinculadas a dimensiones propias de la enseñanza y si estas son de naturaleza similar o cambiante. Asimismo, parece necesario explorar desde la perspectiva de los propios docentes cuáles son las estrategias de inserción y apoyo que utilizan los establecimientos escolares y la manera en que interactúan con sus procesos de iniciación a la enseñanza.

\section{PROPÓSITOS Y METODOLOGÍA}

Tal como fuera expuesto, este artículo presenta parte de los hallazgos generados en dos investigaciones realizadas articuladamente, siendo parte de una línea de investigación desarrollada en torno a los procesos de iniciación a la enseñanza. La primera de ellas tuvo como propósito comprender de qué manera varían las preocupaciones en torno a la enseñanza que experimentan profesores principiantes de Educación Básica; de allí emerge el interés por estudiar los contextos escolares y el rol que cumplen en esta inserción. El segundo estudio aborda, entre otros aspectos, la caracterización de los contextos 
escolares de iniciación desde la perspectiva de profesores principiantes ${ }^{2}$. Ambos adhieren a un enfoque cualitativo y utilizan un diseño de Estudio de Casos (Stake, 1999), tanto por su naturaleza exploratoria como por el interés de analizar y describir a partir de los propios actores la diversidad de maneras en que se expresan estos fenómenos.

Se utiliza un muestreo intencionado cuyo propósito es determinar una muestra heterogénea en relación al contexto escolar de inserción y programa de formación inicial cursado por los participantes. A la vez, se determina un criterio de homogeneidad en cuanto al tiempo de experiencia profesional que posee cada uno de los profesores que participó del estudio, el cual fluctúa entre un año y medio y dos años. El primer estudio se configura con la participación de 12 casos de profesores egresados de seis diferentes universidades ubicadas en la Región Metropolitana con niveles altos, medios y bajos de selectividad. Por su parte, el segundo estudio trabaja con 12 profesores que egresaron de 4 universidades ( 2 ubicadas en la Región Metropolitana y 2 en ubicadas en la quinta Región) cada una con distinto nivel de selectividad.

Como estrategia de recolección de información se utiliza la "entrevista episódica" (Flick, 2000), instrumento semiestructurado, fundamentado en la idea de que las experiencias de los sujetos se almacenan y recuerdan en la forma de un conocimiento de tipo narrativo-episódico y de tipo semántico. El conocimiento episódico se vincula a situaciones y circunstancias concretas, y el conocimiento semántico se constituye a partir de supuestos y relaciones entre esas experiencias que se abstraen y se generalizan. Su objetivo es indagar en las dificultades vividas, en el ámbito de la enseñanza, por profesores y profesoras principiantes de Educación Básica, profundizando sobre los motivos, condiciones y contextos que se relacionan a estas dificultades. El procedimiento de análisis adoptó los principios de la Teoría Fundamentada, la cual puede ser definida como "un conjunto de pautas analíticas flexibles que permiten a los investigadores concentrar su recolección de datos y elaborar teorías inductivas de alcance medio a través de sucesivos niveles de análisis de datos y de desarrollo conceptual" (Charmaz, 2013: 271). Cabe señalar que avanzar hacia una comprensión conceptual requiere que el investigador se interese por la relación entre procesos y estructuras que subyacen a los fenómenos que estudia. En este sentido, referirse a los procesos implica comprender las estrategias, acciones e interacciones de los actores y la significación que le dan a éstos. Por su parte, la dimensión estructural, o condicional, de un fenómeno está dada por los contextos y las condiciones sociales, culturales y de recursos, que influyen, promueven, coartan, facilitan u obstaculizan las acciones e interacciones de los actores (Cisternas, 2017). El análisis se realizó con apoyo de software (Atlas.ti) e involucró codificación abierta y un reordenamiento de categorías en torno a propiedades y dimensiones, comparando similitudes y diferencias entre casos, incidentes y categorías, con el objetivo de descubrir patrones y relaciones en torno al fenómeno central que se está indagando (Strauss \& Corbin, 2002; Glaser 2004). Este sistema de análisis se ajusta de manera pertinente a los objetivos de esta investigación, ya que atiende a su condición de estudio exploratorio.

Esta investigación comprende además el análisis de las prácticas de enseñanza de estos profesores, incidentes críticos vinculados a sus decisiones pedagógicas y didácticas, estrategias personales para enfrentar los desafíos de la iniciación (Fondecyt de Iniciación $N^{\circ} 11130279$ ). 


\section{HALLAZGOS}

A continuación se presentan los resultados del análisis dividido en dos partes. En primer lugar, se caracterizan las dificultades experimentadas por los docentes principiantes de Educación Básica en el ámbito de las tareas propias de la enseñanza, en la búsqueda de reflejar la diversidad de dilemas y su relación con algunos elementos de la formación inicial recibida. En la segunda parte, ponemos la mirada sobre los contextos escolares de iniciación y cómo estos son descritos por los propios docentes noveles. En un análisis que busca reconocer formas de acompañarlos en este proceso, se destacan rasgos de la escuela que pueden hacer más o menos conflictivo y problemático los inicios en la enseñanza y el trabajo docente.

\subsection{DIFICULTADES EN TORNO A LA ENSEÑANZA: HETEROGENEIDAD Y COMPLEJIDAD}

La indagación buscó situar las dificultades desglosadas en tres ámbitos o momentos clave del proceso de enseñar. En primer lugar, se dirige la atención sobre las tareas involucradas en anticipar y diseñar las estrategias y actividades de enseñanza en el marco del currículo escolar, las exigencias de la institución educativa y las necesidades de aprendizaje de los estudiantes (preparación de la enseñanza). En este dominio, se plantea la importancia dada a que el profesor adquiera un profundo conocimiento de su disciplina y de las herramientas pedagógicas que faciliten y adecúen la preparación de su enseñanza al contexto de aprendizaje específico de sus estudiantes, en la perspectiva de comprometer a todos sus estudiantes con los aprendizajes. En segundo lugar, la implementación de estrategias de enseñanza hace referencia a la movilización de todos los aspectos involucrados en el proceso de enseñanza que favorezcan el compromiso real de los estudiantes con sus aprendizajes. Los criterios que componen este dominio apuntan a la misión esencial de la escuela y el profesor: la de generar oportunidades de aprendizaje y desarrollo para todos sus estudiantes. Finalmente, interesa analizar las dificultades en torno a la evaluación de los aprendizajes, la que implica el monitoreo del proceso de comprensión y apropiación de los contenidos por parte de los estudiantes, a fin de retroalimentar tantos sus avances como las estrategias pedagógicas utilizadas por el profesor (Mineduc, 2008). Como se verá a continuación, la visión de los docentes refleja una diversidad en las características de los conflictos y dificultades que describen en su experiencia vinculada directamente al quehacer en el aula.

En cuanto a la preparación de la enseñanza, llama la atención que sea la dimensión que inquieta en menor medida a los docentes. Dentro de los profesores participantes, mayoritariamente se la asocia a una tarea mecánica, burocrática o simplemente tediosa, y en menor medida es planteada como un momento exigente en términos profesionales o sobre el cual no se sienten preparados lo suficientemente. Asimismo, al contrastar estas visiones con las características de los establecimientos escolares donde se desempeñan los docentes, aparece una relación entre aquellas escuelas que suprimen la responsabilidad de planificar, delegándola en otros actores o medios con la escasa preocupación que muestran los docentes principiantes por estas tareas: por ejemplo, en establecimientos donde, producto de asistencias o asesorías técnicas, se entregan planificaciones diarias o la adquisición de libros o programas informáticos que apoyan estos procesos. Por otra parte, pensar la enseñanza antes de entrar al aula emerge como un proceso relevante, especialmente en 
docentes de establecimientos que promueven y dan espacio para planificar individual o colectivamente.

Planificar no me ha tocado todavía. A mí me entregan las planificaciones hechas clase a clase, así todo, así un libro de ancho (Profesora Marisol).

Yo paso muchas horas planificando, por ejemplo, en ciencias, buscando el video, o el documental que diga exactamente lo que yo quiero que diga, porque el tiempo de planificación versus horas de aula, es de tres a uno, entonces tres horas por cada hora de aula (...) y eso si me lo dio la universidad, o sea me dio la habilidad de preparar cada contenido, investigar, tratar de llegar lo más experto posible a cada clase (Profesor César).

En ese contexto, se puede constatar cómo las dificultades presentan una fluctuación que las hace diferentes entre sí. Algunos docentes perciben debilidades en su capacidad para escribir y "volcar" en el papel un diseño de las estrategias de enseñanza.

[...] no es fácil, sobre todo cuando una persona le cuesta la redacción, por ejemplo, o le cuesta pensar en actividades, es como difícil proyectarse un mes, pero eso también lo va adquiriendo uno a través de la misma práctica, los primeros años se hace súper difícil porque uno no sabe qué es lo que tiene que poner, en qué se tiene que fijar (Profesora Patricia).

En otros docentes lo difícil está en la realización de estas tareas siguiendo las indicaciones del establecimiento y sin que éstas se conviertan finalmente en un trámite burocrático que demanda tiempos que, además, son escasos.

Siempre la planificación se hace, más que dificultosa, tediosa, y cada colegio tiene su propia exigencia al respecto, (...), hay otros colegios que son bastante quisquillosos con el tema de la planificación y rebuscados. Entonces uno se lleva mucho trabajo para la casa, demasiado y eso en el fondo se convierte en una dificultad porque pierde uno mucho tiempo (...) y para mí por lo menos me resulta mucho más valioso el tiempo en preparar una guía, un clase bien hecha, una prueba bien hecha (Profesora Paola).

Para algunos estas tareas suponen un esfuerzo adicional cuando se trata de contenidos que no se manejan lo suficiente.

Uno tiene que hacer planificaciones para distintos subsectores, y en los subsectores uno tiene que tener conocimientos del tema, y eso es lo que a mí me complicó, porque yo me especialicé en lenguaje, entonces me sentía, más desventajada (Profesora Ana).

Por otra parte, hay docentes que viven con preocupación la tensión de tener que planificar para aulas muy numerosas y con diversos niveles de aprendizaje y, al mismo tiempo, responder a la exigencia que hacen las instituciones escolares de realizar planificaciones homogéneas.

[...] uno de repente pide a UTP planificar en forma... no sé si 'diferenciada' es la palabra adecuada, pero yo me he dado cuenta que los tres quintos se manejan muy distinto, y que los tres 
sextos básicos son muy distintos unos de otros, hay un sexto que avanza muy rápido, y los otros dos que son más lentos... pero pese a eso no puedes planificar distinto, ;me piden que sea la misma actividad, la misma prueba! Entonces en eso no hay apoyo y se hace difícil (Profesora Marta).

El siguiente esquema da cuenta de las diversas tareas asociadas a la preparación de la enseñanza sobre la que los docentes manifiestan preocupación.

Figura 1: Diversidad de preocupaciones asociadas a la Preparación de la Enseñanza

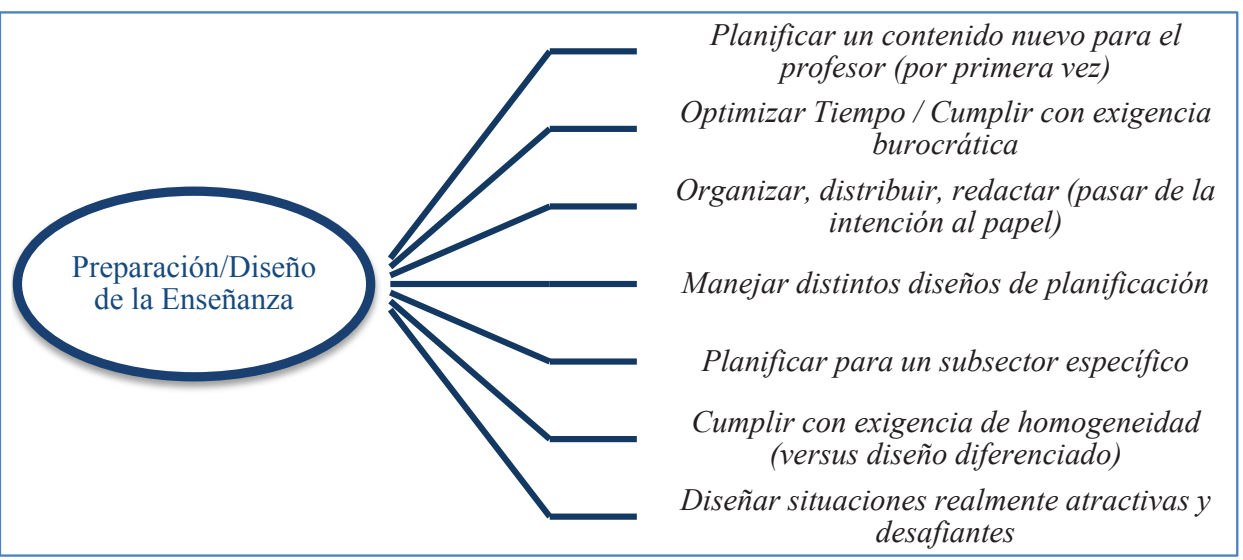

La figura precedente ilustra diferencias significativas en la naturaleza y complejidad de los problemas que se visualizan. Pareciera ser que algunos están vinculados más directamente a capacidades que la formación inicial debiese desarrollar (como el manejo de contenidos o la dificultad para adaptarse a diversos formatos de planificación) y que al expresarse en dificultades estarían dando cuenta de vacíos en este proceso. Mientras que otras podrían ser asociadas a desafíos propios del contexto escolar, como puede verse en la pugna de planificaciones homogéneas o flexibles ante las diferencias de cada grupo de estudiantes.

El segundo grupo de dificultades asociadas a la implementación de estrategias de enseñanza también refleja necesidades de distinto tipo. Algunas derivadas de vacíos o debilidades conceptuales de la formación inicial (como por ejemplo, enseñar contenidos específicos que no se manejan) o bien dilemas más prácticos asociados a la gestión y distribución del tiempo de la clase o el manejo del comportamiento y la participación de los estudiantes.

[...] en matemáticas es difícil. Yo paso los contenidos porque los tengo que pasar, y trato de hacerlo lo mejor posible, pero también con un poquito de inseguridad de que porque como yo soy mala quizá puedo transmitirles eso a los niños. Eso es lo que me da miedo, lo que no me pasa en Lenguaje o en las otras asignaturas (Profesora Estela).

Particularmente en aulas numerosas o en establecimientos que no seleccionan y atienden a una población escolar con altos niveles de vulnerabilidad socioeconómica. 
[...] cómo lidiar, como tratar, como llegar a los niños con dificultad de aprendizaje. Porque a uno no le enseñan en la universidad no le entregan las herramientas, y uno tiene un universo de cuarenta y tantos niños donde diez tienen ese tipo de dificultad y donde obviamente que la clase y cualquier tipo de cosa que uno pretende hacer se dificulta el doble el triple, cuando uno tiene que lidiar con esos niños y en toda mi experiencia laboral he tenido y me he encontrado siempre con ello (Profesora Paula).

También hay quienes sienten inseguridad en situaciones donde creen necesario contar con un repertorio de estrategias diversificadas para enseñar el mismo contenido (si no me resultó enseñándolo de este modo ¿de qué otra forma puedo hacerlo?). En la figura 2 se detalla el resto de preocupaciones que describen los docentes. Cabe destacar cómo algunas están fuertemente mediadas por las demandas del contexto escolar y sus particularidades, otras revelan una convicción acerca del aprendizaje como un fenómeno que involucra los saberes de los estudiantes y un reconocimiento a la importancia del manejo conceptual de aquello que se enseña.

Figura 2: Diversidad de preocupaciones en la fase interactiva Interacción Didáctica)

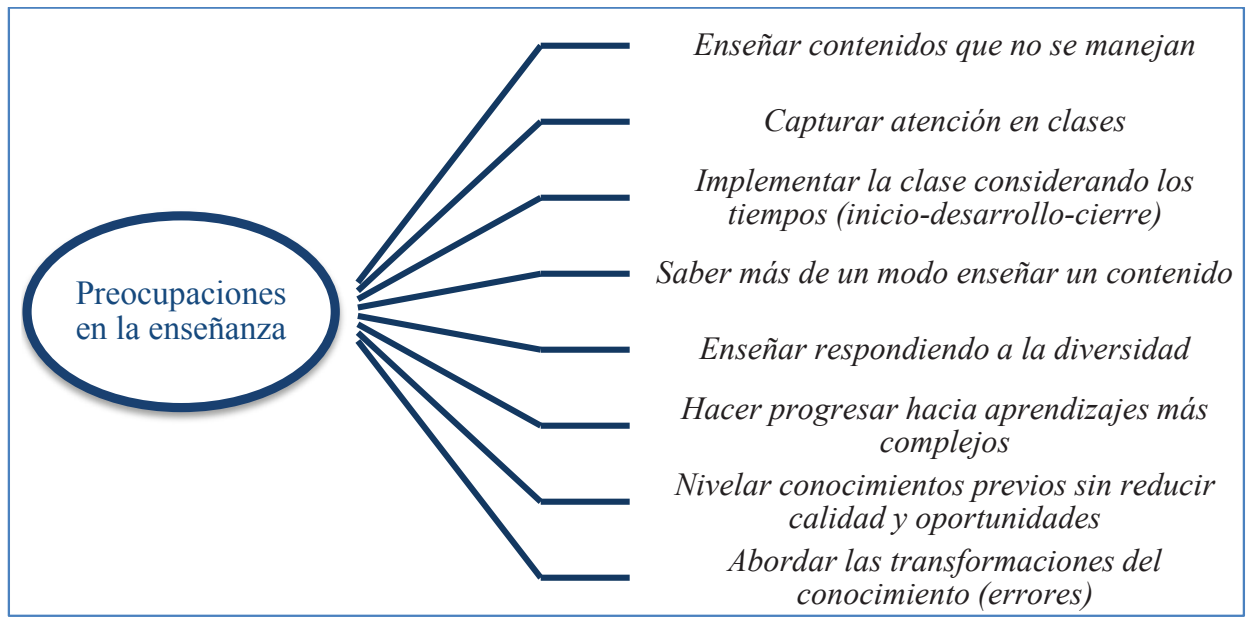

El tercer grupo de preocupaciones que fueron analizadas se sitúa en los procesos de evaluación del aprendizaje. A diferencia de la preparación de la enseñanza, este foco está fuerte y transversalmente presente entre los profesores participantes. ¿Cómo evaluar respondiendo a las necesidades de retroalimentación y a la vez a la demanda por calificar y certificar aprendizajes?

Las mayores preocupaciones se concentran en la construcción de instrumentos pertinentes y útiles para informar tanto al establecimiento como al propio docente de los avances de los niños.

Los indicadores, todas esas cosas, como bajar esa información me costó, y yo creo que es algo que todavía me cuesta, pero, ya, lo sé, más o menos sé ordenar, lo que yo quiero evaluar, 
Estudios Pedagógicos XLII, N 4: 31-48, 2016

PROFESORES PRINCIPIANTES DE EDUCACIÓN BÁSICA: DIFICULTADES DE LA ENSEÑANZA EN CONTEXTOS ESCOLARES DIVERSOS

lo que se está hablando, no, ponte tú, en las mismas pruebas, o sea uno de repente realiza un instrumento que no tiene nada que ver con lo que uno enseñó, entonces eso es, o sea, he sido consciente de que me cuesta y pido ayuda; y es algo que todavía yo siento que estoy coja, no es algo que he logrado superar (Profesora Ana).

[...] no me resulta una prueba, eso es como lo más concreto, no me resulta una prueba, porque copian, ¿qué otra manera tengo para evaluarlos?, porque pueden haber otras maneras de evaluarlos, de hecho en el camino me puse a pensar en eso, porque estos niños no estudian entonces en las pruebas ¿qué hacen? copian ¿qué estrategia puedo ocupar? ¿pero qué hago, que hago con este curso, que hago?, tal vez se me ocurre llamarlos a la pizarra y que me hagan un ejercicio en la pizarra y pero eso se me ocurre a mí, y no sé si sea correcto o no, y no es algo que tu digas eso lo aprendí en la universidad... (Profesora Noelia).

Otras dificultades están asociadas a la relación entre evaluación sumativa y formativa. Los docentes buscan encontrar un equilibrio entre ambas, respondiendo a una concepción de evaluación que vaya más allá de la calificación y que les permita recoger evidencias sobre el proceso de aprendizaje.

Entonces cuando llegué a llegué con las cajitas, me da risa, porque entré al séptimo, y todos los niños me decían ¿y eso para qué? Pero siento que ahí si evalué el proceso, pero fue tedioso la revisada... Entonces claro, eso hace pensar que o yo lo estoy haciendo mal, o la evaluación procesual es demasiado compleja para aplicarla así (Profesor César).

[...] yo tomaba una prueba de nivel, una prueba por unidad, y pare de contar... y los trabajos que podía hacer, pero me di cuenta que de repente uno no tomaba en cuenta el proceso del estudiante... entonces empecé el año haciendo controles periódicos, y eso es cansador: lo controles, la co-evaluación, es todo un show... (Profesora Manuela).

También se presentan dilemas respecto a una evaluación que sea diferenciada, es decir, acorde a los niveles de aprendizaje de sus estudiantes.

P: La evaluación... yo creo que lo más difícil es la evaluación diferenciada; bueno siento yo que es la más difícil, porque yo tengo muchos niños en este instante, que ocupan evaluación diferenciada, y tengo que ocuparme del resto del curso y además ellos. El tiempo de construir una evaluación, me hago el tiempo pero no lo tengo, tengo que ocupar tiempo familiar para construir instrumentos de evaluación diferenciados para ellos, y yo creo que el gran problema es la cantidad de niños por sala, uno no puede hacer una clase exclusiva, para cada... no exclusiva, sino que más personalizada, porque uno tiene que llevar a la masa, porque si no sería darles instrucciones uno por uno, y uno da las instrucciones para todos, y todos entienden de distintas formas, tenemos que unificar criterios, entonces, es difícil... (Profesora Nadia).

Como en las anteriores dimensiones se observan diferencias en la naturaleza o tipo de dificultad. Algunas refieren a aprendizajes más elementales, vinculados a aprendizajes que debiesen ser producto de la formación inicial y otras demandan mayor nivel de especificidad y articulación de saberes y prácticas. 
Figura 3: Diversidad de preocupaciones en torno a la evaluación de aprendizajes.

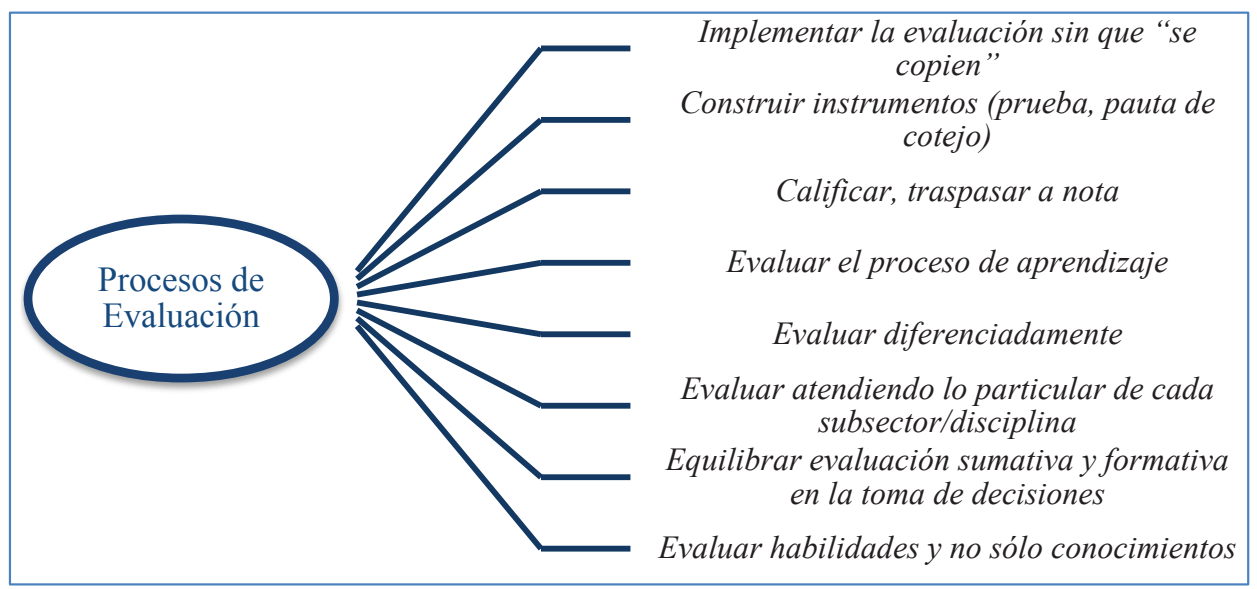

Además de las tres dimensiones precedentes, los docentes dan cuenta de una dificultad más amplia, asociada a la enseñanza de un subsector de aprendizaje específico y que cruza sus decisiones desde la preparación de la clase hasta la evaluación de ésta. Frases como: me cuesta todo lo que sea de matemáticas, revelan Esto se observa principalmente en los profesores principiantes que se formaron con una especialidad (Matemática, Lenguaje y Comunicación, Ciencias, Historia) pero que se insertan en contextos escolares donde deben desempeñarse como profesores generalistas, responsables de la enseñanza de varios subsectores que desconocen con la profundidad suficiente (Me especialicé en Lenguaje y Comunicación y me tienen enseñando matemáticas y ciencias). También resulta una dificultad significativa cuando los docentes son asignados a cursos de segundo ciclo de educación básica y en áreas curriculares que no coinciden con la especialidad.

\subsection{CONTEXTOS ESCOLARES QUE FACILITAN U OBSTACULIZAN LA INICIACIÓN}

Los docentes describen sus entornos laborales desde el punto de vista de las características de sus estudiantes, colegas, directivos, las culturas y prácticas institucionales y roles, tareas y apoyos que les dio (o no les dio) considerando su condición de principiante. El análisis de los casos muestra que los contextos marcan diferencias sustantivas en la calidad de los procesos de inducción profesional y la identidad profesional que van construyendo los profesores. 
Estudios Pedagógicos XLII, N 4: 31-48, 2016

PROFESORES PRINCIPIANTES DE EDUCACIÓN BÁSICA: DIFICULTADES DE LA ENSEÑANZA EN CONTEXTOS ESCOLARES DIVERSOS

Figura 4: Ecologías escolares que facilitan y obstaculizan la inserción del profesor principiante.

\begin{tabular}{|c|c|c|}
\hline & Autoritarismo y presiones de directivos \\
\hline & Pxigencias institucionales sin apoyo (novel como "experto") \\
\hline
\end{tabular}

\begin{tabular}{|c|c|}
\hline & Apoyo y reconocimiento institucional a condición novel \\
\hline \multirow{4}{*}{ 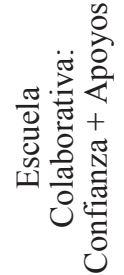 } & Directivos destacados por manejo pedagógico y didáctico \\
\hline & Visitas aula + Retroalimentación "propositiva" \\
\hline & Cultura colaborativa entre pares \\
\hline & Promueve indagación/diseño \\
\hline & Universidad = Escuela sintonía de enfoques pedagógicos \\
\hline
\end{tabular}

Entre los escenarios que obstaculizan los profesores principiantes retratan un tipo de establecimiento escolar que exige, pero no entrega apoyos. Por ejemplo, donde las horas no lectivas no se respetan, no se impulsa el trabajo entre profesores de un mismo nivel o subsector y habitualmente se utiliza al profesor novel como un profesional "comodín", destinado a cubrir inasistencias, reemplazos o cursos conflictivos. Además, los cursos muy numerosos y la falta de apoyos en recursos humanos y materiales son también un factor que provoca mayores preocupaciones y dilemas.

[...] en este colegio uno trabaja bajo presión, hay colegios que realmente son, no se si casi es que son muy... no sé cómo... es bien complejo, es muy exigente entonces uno trabaja bajo presión (Profesora Marta).

\section{¿Hubo algún tipo de apoyo específico por tu condición de profesora novel?}

No, lo único que hicieron fue explicarme un poquito más lo del libro de clases que obviamente no en todos los colegios es de la misma manera, me dijeron cómo tenía que llenar el leccionario, la parte de la asistencia que era como lo que más les preocupa (...) y me tiraron al tiro a los leones, a un cuarto básico, que en realidad es lo que les preocupa a ellos, por el SIMCE, pero me dijeron que saben que me han saturado con cosas, me han visto que yo llego pálida o con las media ojeras, y me han dicho qué te pasa que tienes una cara y yo digo estoy muerta de cansada. El jefe de UTP Me dijo 'yo a las profesoras que son buenas les tengo que dar más horas', así me lo dijo (Profesora Solange). 
Del equipo directivo no puedo decir que fue un apoyo ciento por ciento, porque muchas cosas de las que gestioné fueron por la mía o por la misma profesora con la que trabajo. Al solicitar ayuda. Por ejemplo le digo al jefe de UTP: ¿qué hago en este caso? Los niños todavía me están leyendo muy lento, silábicos algunos ¿Cómo lo hago? Él me contesta: Déjame consulta y te digo. Entonces tengo que buscar por la mí, preguntar en todas partes... (Profesora Marisol).

Otras experiencias en cambio, describen entornos laborales en los que se confía en la capacidad del profesor novel, pero a la vez los orientan para resolver los problemas que emergen en el camino. A diferencia de una escuela que presiona sin ayudas, aquí los establecimientos se caracterizan por el marcado apoyo que reciben todos los docentes y en particular los noveles, asumiendo la mayor demanda de tiempo, contención y retroalimentación que requieren. Destaca la colaboración entre pares, los espacios definidos para el trabajo entre docentes del mismo nivel y el apoyo a la enseñanza mediante prácticas de observación-retroalimentación. La siguiente profesora compara una experiencia de algunos meses con su trabajo actual, durante su primer año laboral:

D: En el colegio XX me tiraron a los leones al tiro: jefatura, inglés, cero posibilidades. En este, ahora, a algunas las pusieron de co-teacher, planificamos todo juntas, tenemos tiempo para eso. Lo que sí nos pedían mucho es que teníamos que tener ideas nuevas. Más creatividad, cómo hacer las clases. Te exigen más en ese sentido por ser más chicas, porque saliste hace tan poco, que tienes que tener ideas frescas (Profesora María Soledad).

También encontramos, aunque escasas, experiencias concretas de mentoría implementadas por establecimientos escolares. El efecto de estos apoyos sobre los docentes es evidente:

El colegio qué hace, tiene un grupo de profesores mentores que principalmente son profesores mayores... Eran cuatro profesores, Entonces, este grupo, el grupo de mentoría, así se le llamaba. (...) Yo en verdad siempre lo agradecí mucho, porque de alguno u otra forma siempre te decían algo que uno nunca se da cuenta, uno ve la clase de una forma, pero otro profesor la puede ver de otra (Profesora Pamela).

Cabe destacar que estos escenarios (Fig. 5) no se construyen a partir de prácticas aisladas. Al contrario, es la combinación de algunas características facilita u obstaculiza el desarrollo profesional en esta etapa. Así, por ejemplo, un contexto facilitador puede tener altos niveles de diversidad en el aula, pero esto no aparece como un factor desestructurante porque existe una cultura escolar de colaboración con fuerte apoyo pedagógico y didáctico para responder a esa diversidad. Por ello, las denominamos como "ecologías" escolares burocráticas e indiferentes a los desafíos de la iniciación, donde suele existir control y presión sin apoyos, generalmente con un sello en los resultados SIMCE y un esfuerzo por distinguirse y competir dentro de la oferta educativa del sector, mientras otras parecen ser ecologías escolares colaborativas, responsabilizadas ante la iniciación.

Finalmente, el análisis permitió también recoger el modo en que los docentes abordan sus conflictos y preocupaciones, especialmente durante el primer año de iniciación. ¿Cómo se sobrevive a los primeros años de inducción a la docencia? Similar a otros estudios (Ávalos, 2005; Flores 2008), los principiantes recurren a una amplia gama de estrategias. 
Sin embargo, observamos que las estrategias son diferentes según la institución y formación inicial que recibieron estos profesores.

En efecto, los datos muestran que la adaptación rápida y "sumisa" a lo que dispone el establecimiento, la reproducción de prácticas que otros docentes de más experiencia recomiendan o la imitación directa de lo que observan en los colegas, son modos habituales en profesores principiantes que se formaron en instituciones que entregan una formación de tipo generalista, registran puntajes muy bajos de selección y presentaron una formación excesivamente teórica, a juicio de los propios docentes. Por el contrario, quienes egresan de instituciones con mejores índices de calidad y vincularon la formación a contextos y prácticas auténticas, utilizan estrategias como comparar lo que hacen varios profesores y seleccionar las mejores prácticas, buscar espacios para trabajar colaborativamente con otros profesores o buscar especializarse para ampliar conocimientos didácticos específicos. Son docentes que demuestran cierta autonomía en sus decisiones, que sostienen estar guiándose por sus propias concepciones pedagógicas.

\section{CONCLUSIONES}

De los resultados se levantan dimensiones que ayudan a entender cómo varían las preocupaciones de los docentes principiantes y qué condiciones son las que subyacen a estas variaciones. En efecto, una visión comprensiva del proceso de iniciación e inserción implica considerar en forma dinámica e interactiva elementos vinculados a condiciones y a la propia biografía del debutante, al tipo de aprendizaje y saberes desarrollados durante su formación inicial, y a los roles y tareas asumidos en el marco de culturas escolares diferentes. Coincidiendo con otros autores y trabajos (Ávalos, 2005; Boer, 2011; Cornejo 1999, 2008; Fuentealba 2006; Peralta, 2009; Ruffinelli, 2014; Rufinelli, Cisternas, Córdova \& Figueroa, 2016), esto supone avanzar hacia una visión de la iniciación pedagógica de los docentes más allá de lógicas individuales, lineales y unidimensionales, sino más bien, como procesos interactivos, multidimensionales y dinámicos.

Tal como lo mencionamos en la primera parte de este artículo, gracias a la investigación acerca de las preocupaciones y dificultades de los profesores cuando se inician, sabemos que hay algunas tareas más críticas que otras (Avalos, 2005; González et al., 2005; Ruffinelli, 2014; Ruffinelli, Cisternas, Córdoba \& Figueroa, 2016; Flores, 2014). También sabemos que el desarrollo profesional es idiosincrático, contextual y se alimenta de la subjetividad de cada docente y, por lo tanto, se involucran preocupaciones que pueden ser de naturaleza diferente.

¿Cómo varían las dificultades? Los dilemas sentidos por los principiantes están lejos de ser homogéneos. Al contrario, el lector podrá notar las diferencias significativas que se producen entre los docentes al referirse a las tareas que se les hacen difíciles y sobre las cuales no tienen muchas certezas. Son preguntas y conflictos que muestran distinta "densidad" pedagógica. Algunas pertenecen a un nivel "inicial" del aprendizaje de la docencia (por ejemplo, tener dificultades para adaptarse y usar diversos formatos de planificación, desconocer cómo se enseña un contenido en particular o cómo se diseña un instrumento de evaluación). Otras dificultades, en cambio, muestran mayor densidad y distinciones más complejas sobre el acto de enseñanza (por ejemplo, preocuparse por equilibrar la evaluación de proceso con la de producto, optimizar y gestionar los tiempos 
para responder a la diversidad, preguntarse qué tipo de mediación es más pertinente ante producciones erradas de los estudiantes).

En suma, podemos distinguir entre preocupaciones vinculadas a deudas o vacíos de la formación inicial y otras que corresponden a preocupaciones propias de la complejidad del aula y los contextos laborales, muchas veces difíciles de reproducir en la formación práctica de la universidad.

Más que un patrón evolutivo -que va desde asuntos técnicos y de gestión del aula hacia preguntas sobre el aprendizaje de los estudiantes-, las preocupaciones muestran diferencias en cuanto a su naturaleza, nivel de profundidad y complejidad, diferentes según las dimensiones que ya hemos mencionado. Este es un llamado a pensar en los programas de inducción y mentoría desde una perspectiva contextualizada, diversificada, evitando homogeneizar necesidades.

Figura 5: Dimensiones para comprender las relaciones entre dificultades de la iniciación y el papel de los contextos escolares.

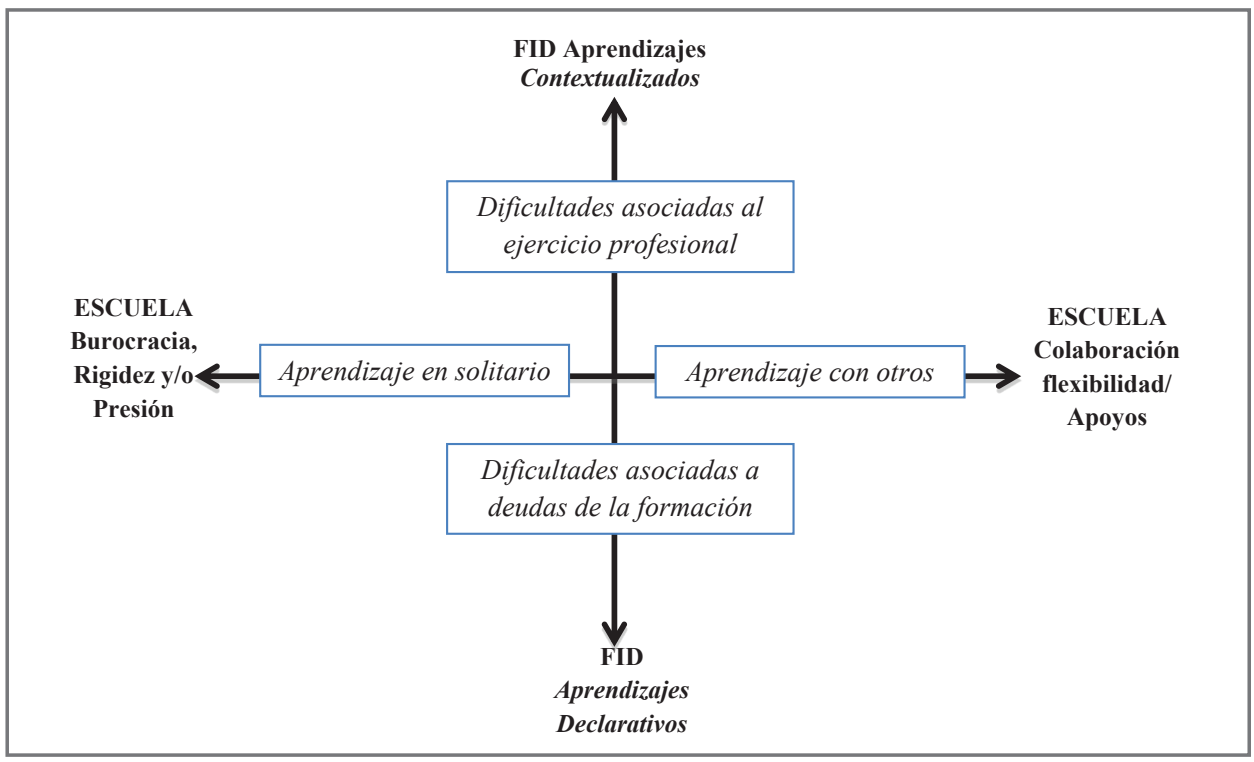

Pudimos ver que la identidad de los docentes se construye especialmente sobre la base de respuestas ante incidentes críticos y un conjunto de representaciones explícitas sobre su rol, la enseñanza y el aprendizaje. De allí la importancia de reconocer las preocupaciones de los docentes y cómo estas varían y se asocian a distintos modos de comprender la profesión y su rol en la escuela.

Estos hallazgos refuerzan la necesidad de contar con investigaciones que ayuden a identificar cuáles son los componentes y las condiciones de los programas de formación que tienen impacto positivo sobre el aprendizaje de la docencia y el desempeño como profesor 
en diversos contextos de enseñanza. Detrás está la comprensión de cómo se produce el desarrollo y aprendizaje profesional y con ello cómo se forjan las identidades docentes.

Finalmente, la proyección de estos hallazgos se puede sintetizar en tres aspectos. En primer lugar, contribuye a mapear las necesidades de apoyo que requieren los profesores principiantes en el contexto chileno y reconocer la homogeneidad o diversidad de estas necesidades en relación a los distintos contextos escolares en los que se insertan los docentes. En segundo lugar, informa sobre buenas prácticas de inducción y apoyos a las prácticas de enseñanza del profesor principiante implementadas por los establecimientos escolares. Y, en tercer lugar, los hallazgos aportan al diseño y revisión de programas específicos de mentoría y apoyo a la inducción de profesores recién egresados y también en la identificación de situaciones problemáticas auténticas que sirvan de base para los programas de formación inicial y continua.

\section{REFERENCIAS BIBLIOGRÁFICAS}

Agüero, C. (2015). Maestras principiantes y la construcción de propuestas didácticas. En R. Menghini \& M. Negrin (Comps.), Docentes principiantes. Aventuras y desventuras de los inicios en la enseñanza. (pp. 159-178). Buenos Aires: Noveduc.

Alliaud, A. (2004). La experiencia escolar de maestros "Inexpertos". Biografías, trayectorias y práctica profesional. Revista Iberoamericana de Educación, 34 (3), 1-11.

Alliaud, A. (2014). Las políticas de desarrollo profesional del profesor principiante en el Programa de Acompañamiento de Docentes Noveles en su Primera Inserción Laboral de Argentina. Revista Brasileira de Educação, 19 (56), 229-242.

Ávalos, B. (2013). El trabajo docente. En B. Ávalos (Ed.), ¿Héroes o Villanos? La profesión docente en Chile (pp. 125-153). Santiago: Editorial Universitaria.

Ávalos, B., \& Matus, C. (2011). La Formación Inicial Docente en Chile desde una Óptica Internacional. Informe Nacional del Estudio Internacional IEA TEDS-M (2010). Santiago: Ministerio de Educación.

Avalos, B., Aylwin, P. y Carlson, B (2005). La inserción laboral de los profesores nuevos en Chile. Paideia 38, 9-27.

Ayala, P., Ortuzar, M., Flores, C., \& Milesi, C. (2015). Búsqueda de empleo, proceso de inserción y dificultad de adaptación de profesores principiantes. Pensamiento Educativo. Revista De Investigación Educacional Latinoamericana, 52 (2), 119-135.

Boerr, I. (2011). (Ed.) Mentores y Nóveles: Historias del Trayecto. Santiago: OEI.

Cisternas, T. (2011). La Investigación sobre Formación Docente en Chile. Territorios Explorados e Inexplorados. Calidad en la Educación, 35 (diciembre), 131-164.

Cisternas, T. (2017). Desafíos y principios para investigar con Teoría Fundamentada. En: Angulo \& Redón (Coords.), Investigación Cualitativa en Educación (pp. 267-278). Buenos Aires: Miño y Dávila.

Cochran-Smith, M., Fries, K., \& Zeichner, K. (2006). Estudio sobre la formación del profesorado en los Estados Unidos: descripción del informe del comité de la American Educational Research Association (AERA) sobre investigación y formación del profesorado. Revista de Educación, 340, 87-116.

Comisión Formación Docente (2005). Informe Comisión sobre Formación Inicial Docente. Santiago, Chile.

Cornejo, J. (1999). Profesores que se inician en la docencia: algunas reflexiones al respecto desde América Latina. Revista Iberoamericana de Educación, (19). Recuperado el 14 de octubre de 2011. 
Correa, J., Martínez-Arbelaiz, A., \& Aberasturi-Apraiz, E. (2015). Post-modern reality shock: Beginning teachers as sojourners in communities of practice. Teaching and Teacher Education, 48, 66-74.

Day, C. \& Gu, Q. (2012) Profesores: Vidas nuevas, verdades antiguas. Una influencia decisiva en la vida de los alumnos. Madrid: Narcea.

Feiman-Nemser (2001). Helping novices learn to teach lessons from an exemplary supporto teacher. Journal of Teacher Education, 52 (1), 17-30.

Flick, U. (2000). Episodic interviewing. En A. M. Bauer \& G. Gaskell (Eds.), Qualitative researching with text, image and sound - a handbook (pp. 75-92). London: Sage.

Flores, A. M. (2008). La investigación sobre los primeros años de enseñanza: Lecturas e Implicaciones. En Carlos Marcelo (Ed.), El profesorado principiante inserción a la docencia (pp. 59-98). Barcelona: Octaedro.

Flores, C. (2014). Inducción de profesores novatos en Chile: un estudio de caso. Pensamiento Educativo. Revista de Investigación Educacional Latinoamericana, 51 (2), 41-55.

Flores, F. (2015). Dificultades laborales de profesores en escuelas secundarias. Educación y Educadores, 18 (3), 411-431.

Fuentealba, R. (2006). Desarrollo profesional docente: un marco comprensivo para la iniciación pedagógica de los profesores principiantes. Foro Educacional, 10, 65-106.

Glaser, B. G. (2004). Remodeling Grounded Theory. Forum Qualitative Social Research, 5 (2). Recuperado el 20 de mayo de 2009, de http://nbn-resolving.de/urn:nbn:de:0114-fqs040245

González, A., Araneda, N., Hernández, J. y Lorca J. (2005). Inducción profesional docente. Estudios Pedagógicos 31 (1), 51-62.

Knoblauch, D., \& Woolfolk, A. (2008). "Maybe I can teach those kids." The influence of contextual factors on student teachers' efficacy beliefs. Teaching and Teacher Education, 24, 166-179.

Lenoir, I., \& Vanhulle, S. (2005). L'état de la recherche au Québec sur la formation à l'enseignement : vers de nouvelles perspectives. Sherbrooke (Canada): Editions du CRP, Université de Sherbrooke.

Marcelo, C. (1988). Profesores principiantes y programas de inducción a la práctica docente. Enseñanza \& Teaching: Revista interuniversitaria de didáctica (6), 61-80.

Marcelo, C. (2008). «Políticas de inserción a la docencia»: De eslabón perdido a puente para el desarrollo profesional docente. En C. Marcelo (Ed.), El profesorado principiante inserción a la docencia (pp. 7-58). Barcelona: Octaedro.

Marcelo, C. (2009). Los comienzos en la docencia: Un profesorado con buenos principios. Profesorado: Revista de currículum y formación del profesorado, 13 (1), 1-25. Recuperado el 14 de octubre de 2011.

Marcelo, C. (2012). Empezar con buen pie: inserción a la enseñanza para profesores principiantes. Olhar de professor, Ponta Grossa, 15 (2), 209-221.

Meckes, L., \& Bascopé, M. (2010). Distribución inequitativa de los nuevos profesores mejor preparados: "Características de origen y destino laboral de los egresados de pedagogía básica". Paper presented at the Primer congreso interdisciplinario de investigación en educación.

MINEDUC (2006). Informe Consejo Asesor Presidencial para la Calidad de la Educación. Santiago, Chile: Ministerio de Educación.

MINEDUC (2008). Marco para la Buena Enseñanza. Santiago, Chile: Ministerio de Educación.

Monereo, C. y Badía, A. (2011) Los heterónimos del docente: Identidad, selfs y enseñanza. En C. Monereo y J. Pozo, La Identidad en Psicología de la Educación: necesidad, utilidad y límites. Madrid: Narcea.

Montecinos, C., Walker, H., Solís, M., Núñez, C., Contreras, I. \& Rittershaussen, S. (2010) Lineamientos para el diseño del currículo del área de Formación de carreras de pedagogía. En: Martinic, S. ¿Fin de ciclo? Cambios en la gobernanza del sistema educativo (pp. 233-255) Santiago: Unesco / Pontificia Universidad Católica. 
Orland-Barak, L. (2008). El impacto de las experiencias prácticas en los profesores principiantes: mentorazgo para formular preguntas diferentes. En C. Marcelo (Ed.), El profesorado principiante inserción a la docencia (pp. 155-176). Barcelona: Octaedro.

Ortúzar, M. S., Flores, C., Milesi, C., \& Cox, C. (2009). Aspectos de la formación inicial docente y su influencia en el rendimiento académico de los alumnos. En Centro De Políticas Públicas (Ed.), Camino al Bicentenario: Propuestas para Chile. Concurso políticas públicas 2009. Santiago: Universidad Católica.

Peralta, L. (2009). Diagnóstico de la inserción profesional de profesores nóveles en escuelas municipalizadas: Tesis Doctoral España.

Ruffnelli, A. (2014). Dificultades de la iniciación docente: ¿iguales para todos?. Estudios pedagógicos, 40(1), 229-242.

Ruffinelli, A., Cisternas, T., Córdoba C., \& Figueroa, J. (2016) Profesores principiantes: una aproximación a la inserción profesional desde la formación, las dificultades y los contextos de iniciación. En: Meckes, L. (Coord.) Políticas y prácticas de Formación de. Profesores en Chile Santiago: CEPPE (En prensa).

Serra, J. (2015) ¿Todos somos principiantes? Reflexiones a partir de las transformaciones en las instituciones de Educación Secundaria. En R. Menghini \& M. Negrin (Comps.), Docentes principiantes. Aventuras y desventuras de los inicios en la enseñanza. (pp. 39-61) Buenos Aires: Noveduc.

Strauss, A. L., \& Corbin, J. (2002). Bases de la investigación cualitativa: técnicas y procedimientos para desarrollar la teoría fundamentada. Medellín: Universidad de Antioquía.

Veenman, S. (1984). Perceived Problems of Beginning Teachers. Review of Educational Research, 54 (2), 143-178.

Vonk, J. H. C. (1983). Problems of the Beginning Teacher. European Journal of Teacher Education, $6(2), 133-150$.

Vonk, J. H. C., \& Schras, G. A. (1987). From Beginning to Experienced Teacher: a study of the professional development of teachers during their first four years of service. European Journal of Teacher Education, 10 (1), 95-110. 